\title{
HIGH GROWTH RATE METAL-ORGANIC MOLECULAR BEAM EPITAXY FOR THE FABRICATION OF GaAs SPACE SOLAR CELLS.
}

\author{
A. Freundlich, F. Newman, C. Monier, S. Street \\ Space Vacuum Epitaxy Center, University of Houston, Houston TX 77204-5507, \\ P. Dargan, M. Levy \\ Riber Inc. 3880 Park Avenue, Edison NJ 08820
}

\section{INTRODUCTION}

The demand for satellite with increased power capabilities has promoted the development and use of high efficiency GaAs-based single junction and multijunction solar cells. Major photovoltaic manufacturers are already producing these devices $[1,2]$ and the technology has captured a large share of the space solar cell market.

Currently high efficiency single junction and multijunction III-V space solar cells are produced by metal-organic chemical vapor deposition (MOCVD). MOCVD requires the use of large quantities of group $V$ hydrides such as arsine and phosphine which raises serious safety and environmental concerns, and also affects the final solar cell cost. Production of such solar cells by Molecular Beam Epitaxy (MBE), which uses solid arsenic and phosphorus species and provides access to a large variety of in situ diagnosis tools, has been proposed to minimize toxicity issues and improve yields $[4,5]$. MBE and related techniques have been shown as being capable of producing very high quality epilayers and devices, including solar cells [5-6]. Recent development of MBE multi-wafers systems capable of MOCVD production throughputs is also an attractive feature. Figure 1 provides a photograph of an MBE Riber 6000 system, the 16" wafer holder in this system could allow the simultaneous growth of III-V on four six inch wafers or $34(6 \mathrm{~cm} \times 4 \mathrm{~cm})$ solar cell wafers per run (Fig.1). Nevertheless, MBE growth rates generally in 1 to1.5 microns per hours range (Growth rates in MOCVD systems ranges from 2-10 $\mu \mathrm{m} / \mathrm{h}$ ) have generated skepticism about the ability of MBE and related techniques as a production tool for thick $(>4 \mu \mathrm{m})$ devices such as solar cells.

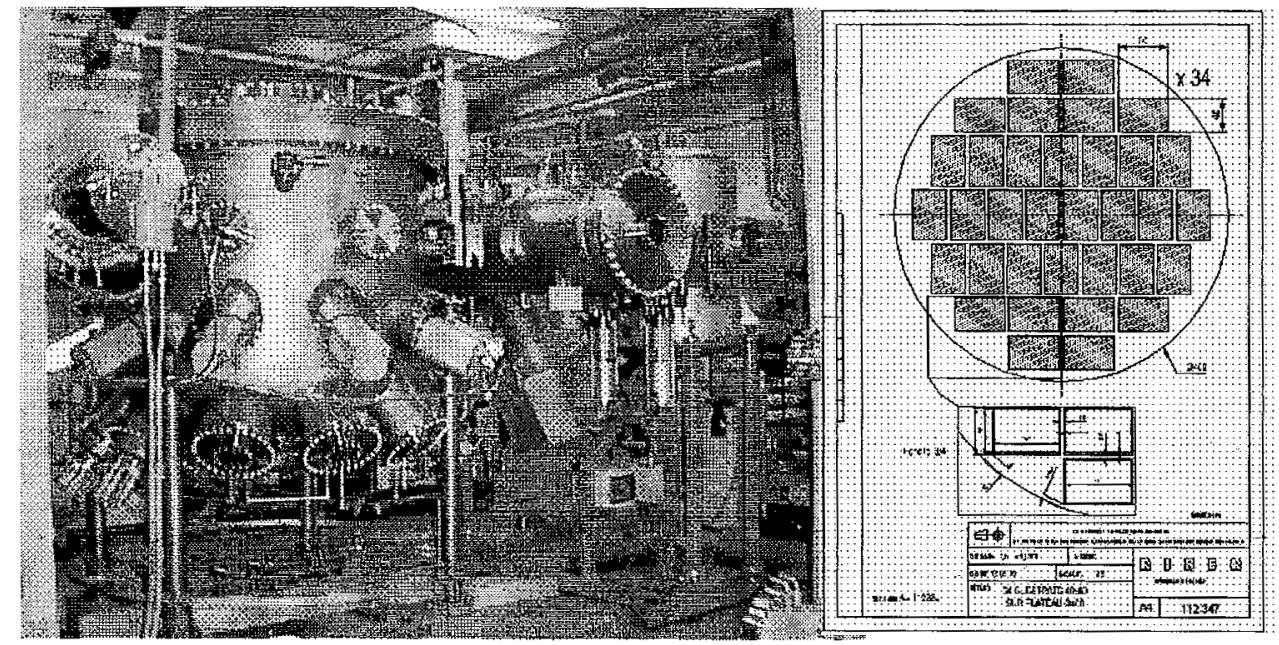

Figure 1: Photograph of a Riber $6000^{\mathrm{TM}}$ MBE production system. Up to $34(40 \mathrm{~mm} \times 60 \mathrm{~mm})$ solar cell wafers can be processed per run. 
In this work it is shown that high quality GaAs photovoltaic devices can be produced by MBE with growth rates comparable to MOCVD through the substitution of group III solid sources by metal-organic compounds. The influence the III/V flux-ratio and growth temperatures in maintaining a two dimensional layer by layer growth mode and achieving high growth rates with low residual background impurities is investigated. Finally subsequent to the study of the optimization of $n$ - and $p$ doping of such high growth rate epilayers, results from a preliminary attempt in the fabrication of GaAs photovoltaic devices such as tunnel diodes and solar cells using the proposed high growth rate approach are reported.

\section{GROWTH AND CHARACTERIZATION}

All growth runs are performed in Riber CBE32 reactor. Tri-ethyl-gallium (TEG) and tri-methylindium (TMI) are used as group III precursors. The organometallics(OM) are maintained at $40 \mathrm{C}$ and introduced mixed with hydrogen through a low temperature $(80 \mathrm{C})$ injector. The OM flux is adjusted using mass flow controllers. To allow an optimum operation of the flow controller the down-stream pressure of the $\mathrm{OM} / \mathrm{H}_{2}$ mixture is maintained at 40 torr using pressure controllers. The group $\mathrm{V}$ element flux was provided by a tetrameric arsenic $\left(\mathrm{As}_{4}\right)$ source. The Arsenic flux at the substrate was between $1.4 \times 10^{-5}$ torr. Si and Be solid sources are used as n-type and p-type dopants respectively.

$\mathrm{GaAs}$ layers are grown with growth rates ranging from 0.5 to 3 monolayers $(\mathrm{ML})$ per second $(0.5-$ 3.2 microns per hour) on nominal (100) highly Si doped GaAs wafers. The GaAs surface reconstruction and growth mode is monitored real- time using reflection high-energy electron diffraction (RHEED). Substrate temperatures are measured using an IR sensitive pyrometer. The precursor decomposition and species partial pressures (flux) in the reactor were monitored using mass spectrometry.

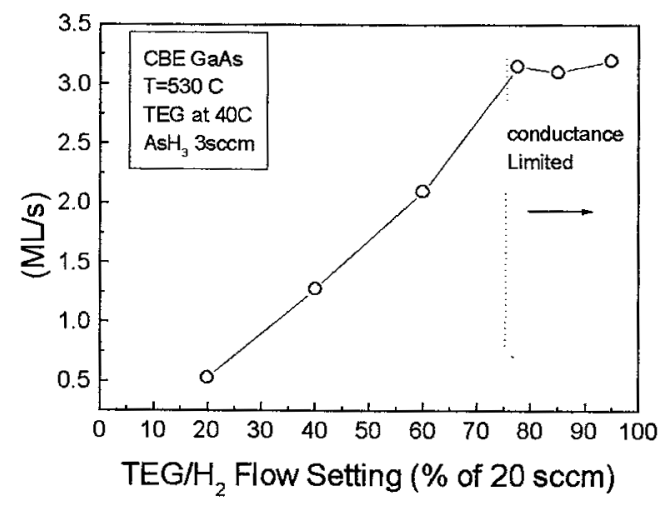

Figure 2: Growth rate as a function of TEG/H2 flux setting.

The GaAs growth rate as determined through the intensity oscillations of the specular beam in the (2x4) reconstruction RHEED diagram is found to increase linearly with the increasing TEG flux. However the growth rate saturates for fluxes exceeding $16 \mathrm{sccm}$ of $T E G / \mathrm{H}_{2}$ mixture to slightly above $3 \mathrm{ML} / \mathrm{sec}$. It is worth noting that beyond this threshold partial and total pressure analysis indicated that the OM pressure in the chamber remained unchanged. The latest suggests that the growth rate is most likely limited by the conductance of our TEG/ $/ \mathrm{H}_{2}{ }_{18}^{1}$ inch-diameter lines.

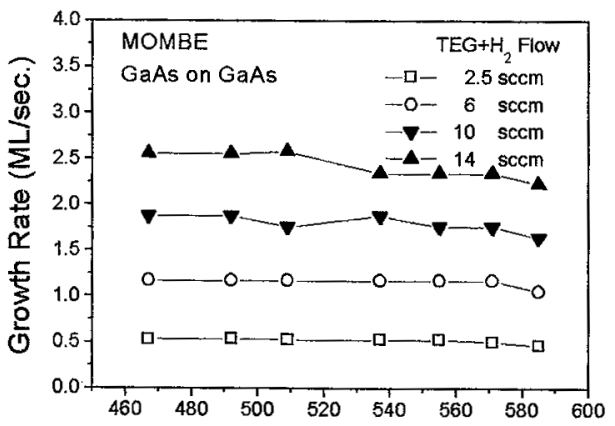

Substrate Temperature (Degrees $C$ )

Figure 3: GaAs growth rate (a) as a function of growth temperature for various TEG fluxes $14 \mathrm{sccm}$ (solid up triangle) $10 \mathrm{sccm}$ (solid down triangle) $6 \mathrm{sccm}$ (open circle) and $2.5 \mathrm{sccm}$ (open square). 


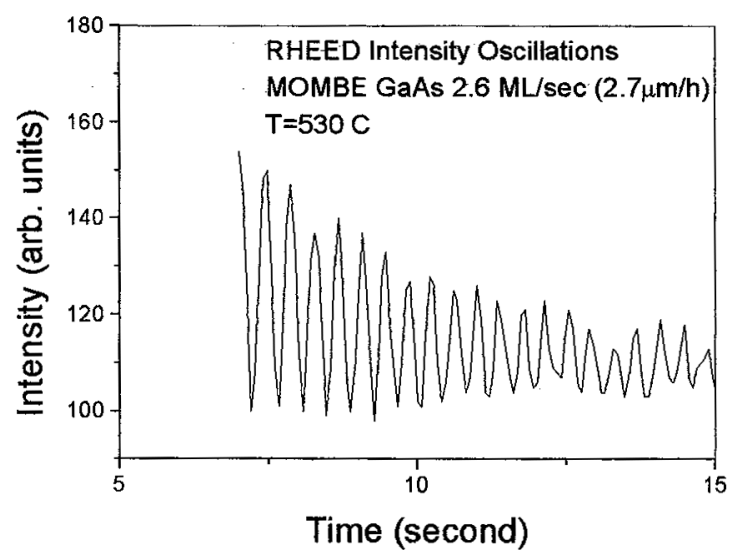

Figure 4: RHEED specular beam intensity oscillations recorded during the growth of GaAs (growth rate $2.62 \mathrm{ML} / \mathrm{sec}$, temperature $530 \mathrm{C}$ )

The MOMBE growth of GaAs is investigated over a wide range of temperature. A 2x4 RHEED diagram and a nearly constant growth rate are obtained within a range of $460-560 \mathrm{C}$ (Figure 3). A slight drop in the growth rate is noticed for temperatures exceeding $570 \mathrm{C}$ suggesting a reduction in the $\mathrm{Ga}$ sticking coefficient. A fuzzy RHEED diagram suggesting the epilayer surface roughening characterized growths performed at substrate temperatures below $500 \mathrm{C}$ or/and with high arsenic partial pressures. Strong RHEED intensity oscillations demonstrating a layer by layer growth mode are observed in the range of 520-580C. In optimum growth conditions even at growth rates exceeding 3 monolayer/sec distinct RHEED intensity oscillations are observed (Figure 4).

GaAs layers grown under optimum conditions (520-590C) exhibit a specular morphology. Background carrier concentrations determined using $\mathrm{C}-\mathrm{V}$ electrochemical profilometry indicate the possibility of obtaining high growth rate GaAs layers with residual active impurity levels in $10 \mathrm{w} 10^{16} \mathrm{~cm}^{-3}$ range. The bound excitonic transitions at $1.512 \mathrm{eV}$ and Carbon neutral acceptor-band (e-C0) at $1.494 \mathrm{eV}$ dominate the 10K-photoluminescence spectra of these samples. As for conventional MBE/CBE samples, these photoluminescence analyses suggests carbon as the main residual acceptor impurity in our high growth rate not intentionally doped epilayers.
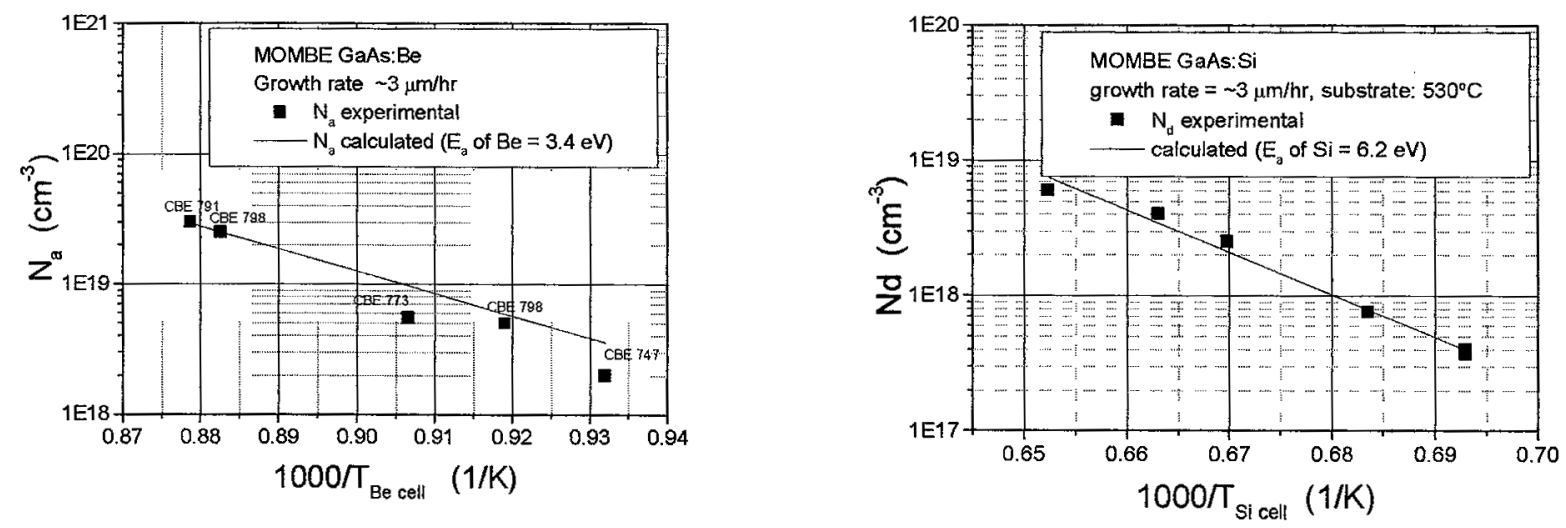

Figure 5: $\mathrm{N}$ and $\mathrm{P}$ type doping of GaAs grown at 3 microns/hour

$n$ and $p$ type intentional doping of GaAs layers grown at 3 micrometer/hour was performed using Be and Si respectively. Intentional beryllium p-type doping ranging from $5 \times 10^{17} \mathrm{~cm}^{-3}$ to $2 \times 10^{20} \mathrm{~cm}^{-3}$ and silicon $n$-type doping ranging from $3 \times 10^{17} \mathrm{~cm}^{-3}$ to $8 \mathrm{x}^{18} \mathrm{~m}^{-3}$ were obtained reproducibly. Doping 
concentrations are compatible with the development of photovoltaic devices such as solar cells and tunnel diodes. The activation energies associated with dopant incorporation in the solid phase are in agreement with those observed for materials grown with lower growth rates and are in the order of $3.4 \mathrm{eV}$ and $6.2 \mathrm{eV}$ for Be and Si respectively (Fig.5).

\section{PRELIMINARY PHOTOVOLTAIC DEVICE PERFORMANCE}

Finally in order to validate the compatibility of the developed high growth rate epilayers for the fabrication of GaAs based advanced solar cells a preliminary attempt is made to fabricate a GaAs $\mathrm{p} / \mathrm{n}$ solar cell and a tunnel diode.

Device structures are grown at rates of about 3 microns /hour on a highly n-doped 3 inch diameter (100) GaAs wafer at a temperature of 530-560 C. Following the growth process non-alloyed $\mathrm{Au}$ metallic layers, deposited by vacuum evaporation, are used as n-type (on substrate) and p-type ohmic contacts. The Au-top contact grid is obtained using a lift off technique.

For the solar cell devices an intermediate highly p-doped GaAs layer is introduced to lower the top contact resistivity. A mesa etching of the structure provides a total area per cell of $5 \times 5 \mathrm{~mm}^{2}$. The topgrid shadowing is estimated to be about $7.5 \%$. The GaAs contact layer was removed from the cell active area using a selective etching and the $\mathrm{Al}_{0.8} \mathrm{Ga}_{0.2} \mathrm{As}$ window layer as an etch stop. Following this step a two layer $\mathrm{MgF}_{2} / \mathrm{ZnS}$ anti-reflection coating is implemented to minimize the solar cell reflective losses. Concerning the tunnel diode device processing a mesa is etched directly using the top contact dots as a mask The latest provides a set of devices with mesa diameters of 100 and 200 microns.

\section{GaAs p/n Tunnel diodes}

Figure 6 shows the C-V profile of the fabricated tunnel diode stressing the high dopant incorporation and the sharpness of the $\mathrm{p} / \mathrm{n}$ interface. All diodes exhibit high peak current densities in excess of $150 \mathrm{Acm}^{-2}$, a good peak to valley ratio and a specific resistivity of less than $0.5 \mathrm{~m} \Omega \mathrm{cm}^{2}$ (at 30 $\left.\mathrm{A} / \mathrm{cm}^{2}\right)$. Current- voltage characteristic of such device are reported in Figure 7 . It is worth nothing that this preliminary tunnel diode device outperforms best MBE-grown GaAs devices[5] $\left(\mathrm{Jp}=46 \mathrm{~A} / \mathrm{cm}^{-2}\right)$ and has a performance comparable to best results achieved by carbon-doped MOCVD tunnel diodes [7]. The associated voltage drop in a tandem device operating using such tunnel diodes even under moderate concentration $(x 20-x 50)$ is expected to be below few $\mathrm{mV}$ and therefore negligible.

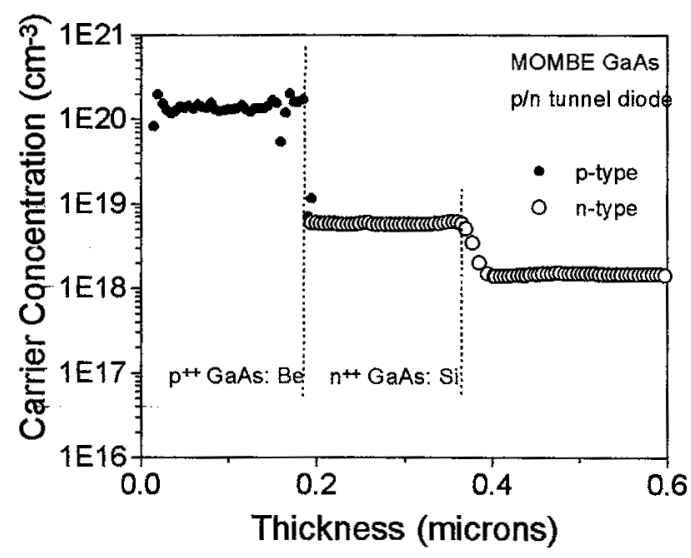

Figure 6: Carrier concentration profile of MOMBE grown GaAs tunnel diode.

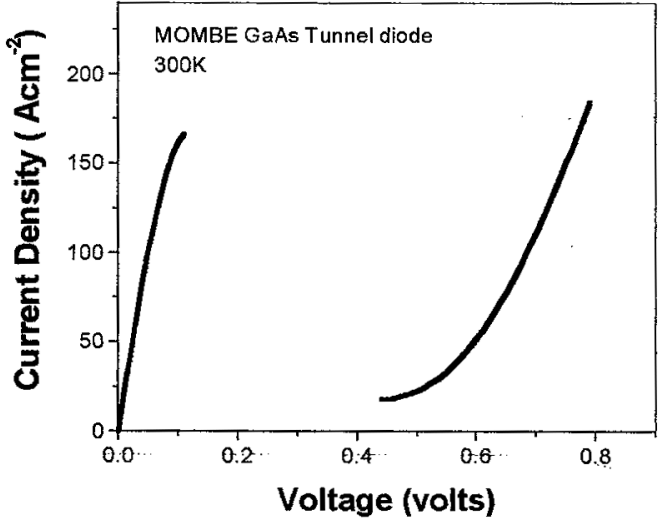

Figure 7: I-V characteristics of a 100 microns diameter MOMBE GaAs tunnel diode. $\mathrm{Jp}=162$ $\mathrm{A} / \mathrm{cm}^{-2}$

\section{GaAs p/n Solar cell}

All solar devices exhibit good PV characteristics. Room temperature spectral response of this preliminary device is provided in Figure 8 and compares well with data reported in the literature for high quality GaAs MOCVD grown solar cells $[1,2,8]$. The typical device short circuit current exceed $32 \mathrm{~mA} \mathrm{~cm}^{-2}$ 
under one sun AMO simulation with the best device on the wafer reaching $32.5 \mathrm{mAcm}^{-2}$ (comparable to the highest current densities reported for GaAs solar cells). Devices exhibit an open circuit voltage in excess of $950 \mathrm{mV}$, about $5 \%$ below the state of the art GaAs solar cells. Despite poor fill factor 74-78\% (compared to $86-88 \%$ for best GaAs solar cell), probably associated with a non optimized GaAs solar cell design/processing, all cells exhibit AMO efficiencies in excess of $16 \%$. We believe that through the optimization of cell design and processing, cells with efficiencies in excess of $20 \%$ efficient cell would be easily achieved with high growth rate Metal-organic MBE.

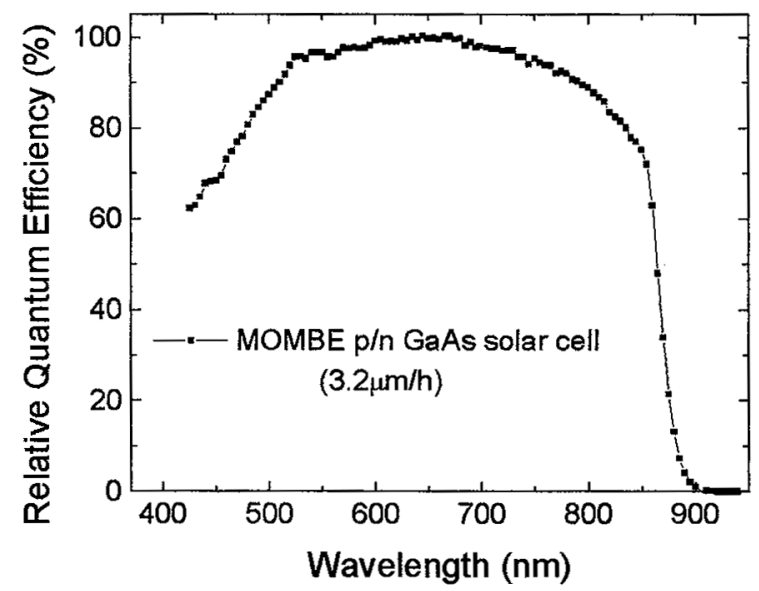

Figure 8: External quantum efficiency of high growth rate MOMBE GaAs p/n solar cell.

\section{CONCLUSION}

In this work we have shown that high quality GaAs epilayers can be produced by MOMBE at growth rates exceeding $3 \mu \mathrm{m} / \mathrm{h}$. Despite high growth rates, the optimization of III/ flux-ratio and growth temperatures leads to a two dimensional layer by layer growth mode characterized by a (2x4) RHEED diagrams and strong intensity oscillations. The material characteristics are shown to be compatible with the fabrication of high efficiency space-photovoltaic devices. As mentioned earlier the growth rates here seems to be limited by the conductance of the TEG delivery line rather than by the growth kinetics. One then may speculate that substantially higher growth rates would be attainable using standard larger diameter $\mathrm{OM}$ delivery line. Preliminary high growth rate GaAs photovoltaic device fabrication has yielded to the realization of record breaking tunnel diodes and encouraging solar cell performance. These results demonstrate the potential of MOMBE as reduced toxicity alternative for the production III-V space photovoltaics. It is worth noting that even at a rate of 3 microns per hour an existing MBE production system would be capable of producing over 100,000 solar cell epi-wafer per year.

\section{AKNOWLEDGEMNET}

The work at University of Houston was partially supported through the NASA Cooperative agreement NCC8-127 and AFRL research grant.

\section{REFERENCES}

1. Y.C.M. Yeh, C.L. Chu, J. Kinger, F.F. Ho, J.M. Olson, M. Timmons, in Proc. $25^{\text {th }}$ IEEE Photovoltaic Specialist Conference, (1996) p187.

2. E.B. Linder, J.P. Hanley in Proc. $25^{\text {th }}$ IEEE Photovoltaic Specialist Conference(1996), p267

3. M. Meyer, R. E. Metzger, Compound Semiconductors (Special Issue), pp 40-41(1997).

4. S. Ringel, R.M. Sieg, S.M. Ting and E. Fitzgerald, Proc. $26^{\text {th }}$ IEEE Photovoltaic Specialist Conference, 1997, p793-799

5. J. Lammasniemi, K. Tappura, R. Jaakola, A. Kazantev, K. Rakennus P.Uusimaa, M. Pessa, in Proc.25 ${ }^{\text {th }}$ IEEE Photovoltaic Specialist Conference, 1996, p97-100

6. A. Freundlich, M. Vilela, A. Bensaoula and N. Medelci, Proc. 23 IEEE PVSC (1993) p 685

7. K.A. Bertness, S.R. Kurtz, D.J. Friedman and J. M. Olson, in Conference records of $1^{\text {st }}$ World Conference on Photovoltaic Energy Conversion (1994) p1859-1862

8. K.A. Bertness, S.R. Kurtz, D.J. Friedman A.E. Kibbler, C. Kramer and J. M. OIson in Conference records of $1^{\text {st }}$ World Conference on Photovoltaic Energy Conversion (1994) pp 1671-1675. ibid Appl. Phys. Lett. 65, (1994) p989 